\title{
Insulin Requirements and Management in Trans-Catheter vs. Surgical Aortic Valve Replacement
}

\author{
Yunyan Qu1 ${ }^{1}$, Liyu Xing², Nazanene H. Esfandiari'1, George M. Deeb², Roma Gianchandani ${ }^{*}$ \\ ${ }^{1}$ Department of Internal Medicine, Michigan Medicine, University of Michigan, Ann Abor, MI, USA \\ ${ }^{2}$ Department of Cardiac Surgery, Michigan Medicine, University of Michigan, Ann Abor, MI, USA \\ Email: *romag@med.umich.edu
}

How to cite this paper: Qu, Y.Y., Xing, L.Y., Esfandiari, N.H., Deeb, G.M. and Gianchandani, R. (2019) Insulin Requirements and Management in Trans-Catheter vs. Surgical Aortic Valve Replacement. Open Journal of Thoracic Surgery, 9, 17-30. https://doi.org/10.4236/ojts.2019.93003

Received: August 9, 2019

Accepted: September 15, 2019

Published: September 18, 2019

Copyright () 2019 by author(s) and Scientific Research Publishing Inc. This work is licensed under the Creative Commons Attribution International License (CC BY 4.0).

http://creativecommons.org/licenses/by/4.0/

\begin{abstract}
Objective: Trans-catheter Aortic Valve Replacement/Aortic Valve Implantation (TAVR) are increasingly performed today. We compared insulin requirements between TAVR and Surgical Aortic Valve Replacement (SAVR) patients with and without diabetes mellitus (DM) to determine optimal glucose management strategies during the perioperative period (POP). Methods: Charts of consecutive patients undergoing aortic procedures were retrospectively reviewed for glucose ranges, insulin requirements and routes of insulin administration (subcutaneous vs. intravenous) for patients with and without $\mathrm{DM}$ to maintain $\mathrm{BG}<180 \mathrm{mg} / \mathrm{dl}$ and compared between the surgical and trans-catheter groups. Results: Patients with SAVRs without DM and A1C < $6.5 \%$, needed low dose insulin infusions and could be transitioned to subcutaneous insulin by 1.5 postoperative days. Patients with SAVR and DM had higher insulin requirements and were transitioned by 2.5 postoperative days. Patients with TAVR with no known DM did not need an insulin infusion in the perioperative period. Patients with TAVR with DM and an HbA1C < $6.5 \%$ did not require insulin infusions during the procedure but only postoperatively for short periods. Patients with $\mathrm{DM}$ and $\mathrm{A} 1 \mathrm{C} \geq 6.5 \%$, required higher doses of insulin infusion and transitioned to subcutaneous insulin by postoperative day 1 . Conclusions: TAVR patients have different insulin requirements compared to SAVRs $(\mathrm{p}<0.05)$. This information helps build a glucose management algorithm for a procedure which is increasingly performed.
\end{abstract}

\section{Keywords}

Aortic Stenosis, Diabetes Mellitus, Trans-Catheter Aortic Valve Replacement, Surgical Aortic Valve Replacement, Blood Glucose Management 


\section{Background}

Aortic Stenosis (AS) is a common valvular disorder with as many as 300,000 people in the United States diagnosed with severe AS each year [1]. After the onset of symptoms and without aortic valve replacement, patients have a survival rate of $50 \%$ at 2 years and $20 \%$ at 5 years [2].

Surgical aortic valve replacement (SAVR) with an open heart procedure was the treatment of choice for severe AS in the past. However, approximately one-third of AS patients are elderly and are high-risk with significant co-morbidities [1]. Less invasive catheter-based alternative is, therefore, sought for this high-risk population, including The Core Valve Evolut R system, Transcatheter Aortic Valve Implantation/Transcatheter Aortic Valve Replacement (TAVR). Today, TAVR is first-line therapy for inoperable patients with severe AS and an alternative to SAVR in operable high-risk patients.

Hyperglycemia is a physiological stress response and commonly seen in the postoperative setting, particularly after cardiac surgery [3]. Prospective and observational studies have found that hyperglycemia after cardiothoracic surgery in patients with and without diabetes increases morbidity and mortality [4], increases hospital length of stay and costs [5] [6]. Insulin infusion protocols are widely instituted in surgical Intensive Care Units (ICU) to address hyperglycemia in cardiac surgery patients. Currently, there is no perioperative glycemic management protocol or guideline for TAVR patients. From clinical experience, we find that insulin infusion guidelines and protocols for SAVR patients may be too stringent for TAVR patients, considering this procedure is minimally invasive and the majority of the patient population is elderly. Also, since this group does not undergo hypothermic circulatory arrest, significant hyperglycemia is less common. Therefore, blood glucose (BG) management guidelines could be less stringent than for the open heart surgery patient.

We, therefore, designed a retrospective review to compare the TAVR and SAVR patient populations in terms of insulin infusion requirements, infusion length, transition to subcutaneous insulin and hospital length of stay for appropriate BG control. Using this data, we could determine therapeutic guidance for BG management in the TAVR population as there is emerging data on morbidity and mortality outcomes in TAVR patients with diabetes [7] [8] [9] [10]. To our knowledge, this is the first study to address this issue.

\section{Research Design and Methods}

We collected patient data from 2014-2015 by reviewing electronic medical records used at Michigan Medicine (Epic). Data collected include demographic information, BG levels, length of hospitalization and insulin requirements. Surgical data were collected from the surgical database. All cases of AS procedure including SAVR (AVR and Mini invasive-cardio pulmonary bypass procedure) and TAVR were reviewed and assessed for underlying surgical risk based on the 2014 AHA/ACC guidelines, the STS-PROM score. 
1) Inclusion Criteria: Patients over 18 years, with aortic valve replacement as a single procedure or TAVR; with or without a history of diabetes were included for evaluation.

2) Exclusion Criteria: Patients with hemoglobin less than $10 \mathrm{~g} / \mathrm{dl}$ prior procedure, prolonged ventilation more than 24 hours, pressors more than 24 hours, high dose steroids postoperatively, tube feeds post-procedure, liver failure, preand post-operative infections, or on hemodialysis or CRRT were excluded.

In our institution, the hospital glucose management program follows all hyperglycemic patients with SAVR and TAVR during hospitalization. We performed a retrospective review of consecutive inpatient records from 6/2014-12/ 2015. Patients were divided into 4 subgroups; SAVR with DM, SAVR without DM; TAVR with DM and TAVR without DM. The DM groups were further divided into SAVR with DM and HbA1c < 6.5\%, SAVR with DM and HbA1c $\geq 6.5 \%$; TAVR with DM and HbAlc $<6.5 \%$ and TAVR with DM and HbAlc $\geq 6.5 \%$.

3) Blood Glucose Test: Laboratory BG was checked by SIEMENS ADVIA 1800 Chemistry, HGB (Hemoglobin) and Hemoglobin A1c (HbA1c) was checked by The Tosoh G8 HPLC Analyzer/TOSOH BIOSCIENCE) prior to patient admission or prior to surgery. During surgery and perioperatively, BG was measured by the Accu-Chek'-Inform II/Roche Point of Care glucose meters.

On the day of the procedure, oral anti-diabetes agents were held and basal insulin reduced to $70 \%$ the night before the procedure. Meal insulin was held on day of the procedure. During surgery, an insulin infusion was initiated in the operation room (OR) or in ICU. As per the hospital protocol for cardiac surgery ICU patients, an intravenous insulin infusion was initiated when two BG values were over $150 \mathrm{mg} / \mathrm{dl}$ or one was over $200 \mathrm{mg} / \mathrm{dl}$. BG was monitored every hour while the drip was being titrated and reduced to every 2 hours when BG was within therapeutic range (insulin infusion BG titration goal was $110-150 \mathrm{mg} / \mathrm{dl}$ as per the institutional insulin protocol).

Insulin infusions were generally transitioned to subcutaneous insulin when patients were extubated, had weaned off vasopressors, started eating or had relatively stable infusion rates. For TAVR patients who were generally on the step down floors after surgery, the infusion initiation and discontinuation criteria were less protocolized.

4) Statistical Analyses: Statistic analysis performed using Prism 5. Data presented as Mean \pm SEM. One ANOVA and Turkey's multiple comparison test was employed for comparison between groups.

\section{Result}

In this retrospective review of aortic valve procedures, 505 patients were operated. One hundred eighty six patients had a SAVR and 319 had a TAVR procedure. Their demographic characteristics are described in Table 1 including further subgroups of those with and without diabetes. Average age for TAVR patients was 78.4 years, significantly higher than the SAVR group of 68.4 years $(\mathrm{P}<0.05)$. The incidence of $\mathrm{DM}$ in the TAVR group was $44.3 \%$, and 
Table 1. Case component for each group. Data presented as Mean \pm SME for age.

\begin{tabular}{ccccc}
\hline & \multicolumn{2}{c}{ SAVR N $=186$} & \multicolumn{2}{c}{ TAVR N = 319 } \\
\cline { 2 - 5 } Characteristics & $\begin{array}{c}\text { SAVR w/o DM } \\
(\mathrm{n}=128)\end{array}$ & $\begin{array}{c}\text { SAVR w DM } \\
(\mathrm{n}=58)\end{array}$ & $\begin{array}{c}\text { TAVR w/o DM } \\
(\mathrm{n}=173)\end{array}$ & $\begin{array}{c}\text { TAVR w DM } \\
(\mathrm{n}=146)\end{array}$ \\
\hline Percentage (\%) & 68.82 & 31.18 & 44.33 & 45.77 \\
Male (\%) & $87(68)$ & $40(69)$ & $91(53)$ & $83(57)$ \\
Female (\%) & $41(32)$ & $18(31)$ & $82(47)$ & $63(43)$ \\
Age (years) & $64.6 \pm 1.3$ & $69.5 \pm 1.3$ & $78.94 \pm 0.9$ & $77.9 \pm 0.7$ \\
BMI & $28.59 \pm 0.43$ & $31.90 \pm 0.81^{*}$ & $27.19 \pm 0.47$ & $30.89 \pm 0.59^{*}$ \\
HbAlc & $5.49 \pm 0.04$ & $6.85 \pm 0.11^{*}$ & $5.47 \pm 0.04$ & $6.87 \pm 0.10^{*}$ \\
\hline
\end{tabular}

*: Compared with SAVR w/o DM, p $<0.05$. *: Compared with TAVR w/o DM, p $<0.05$.

higher than SAVR group at $31.2 \%$.

Within SAVR group, patient with DM's were older than those without DM $(\mathrm{P}<0.05)$. In the TAVR group, there was no difference in age between patients with or without DM. Patients with DM had a higher BMI and HbAlc than those without DM in both procedures $(\mathrm{P}<0.05)$.

Average operating room (OR) time for TAVR procedure was $4.7 \pm 0.1$ hours, significantly shorter than SAVR time of $7.1 \pm 0.2$ hours $(\mathrm{P}<0.05)$. There were no time differences in OR time between DM patients and non-DM patients respectively in TAVR and SAVR group (Figure 1).

1) Insulin Doses:

During OR and post-procedure, the insulin infusion rates, insulin dosages and infusion lengths were significantly different between the TAVR and SAVR groups respectively. In the OR, TAVR patients needed a lower dose of insulin than the SAVR group. Within the same procedure, patients with DM had higher insulin requirements than those without $\mathrm{DM}$ respectively $(\mathrm{P}<0.05)$ (Figure 2$)$.

On OR day, insulin required by SAVR patients without DM was $24.9 \pm 1.1$ units and $44.3+3.9$ units for those with DM. Patients without diabetes undergoing TAVR required $1.8 \pm 0.3$ units of IV insulin compared to $18.5+1.8$ units for TAVR patients with DM. (Figure $2(\mathrm{a}), \mathrm{P}<0.05)$. Insulin requirements were lower for all days of post-surgery for TAVR procedures (Supplemental Table S1).

Over $90 \%$ of patients undergoing SAVR with DM, required an insulin infusion for the first 3 postoperative days (POD). For SAVR without DM, 84\% required an insulin infusion on POD1 and this percentage was almost half by POD2.

For TAVR patients with DM, $81 \%$ needed an insulin infusion on POD1, and $70 \%$ by day POD 2 and POD3. In contrast, in TAVR without DM only $13 \%$ required an insulin infusion on POD1, and only $3.5 \%$ on POD2 and POD3 (Table 2 ). The dosage of insulin also trended down over the PO days. There was significant difference between insulin requirements between the two procedures 


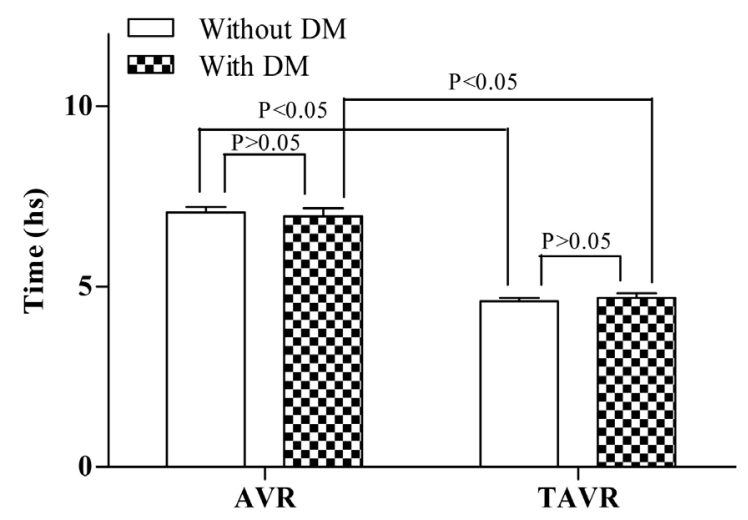

Figure 1. Time in operation room. SAVR w/o DM: $\mathrm{n}=$ 128; SAVR w DM: $\mathrm{n}=58$; TAVR w/o DM: $\mathrm{n}=173$; TAVR w DM: $\mathrm{n}=146$. Data presented as Mean \pm SME. One way ANOV analysis plus Tukey's Multiple Comparison Test performed. $\mathrm{P}$ value presented as in panel.

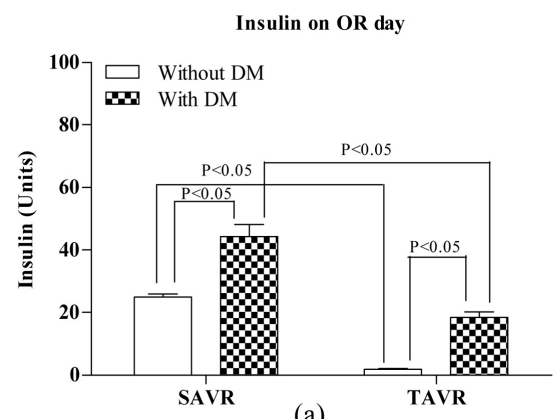

(a)

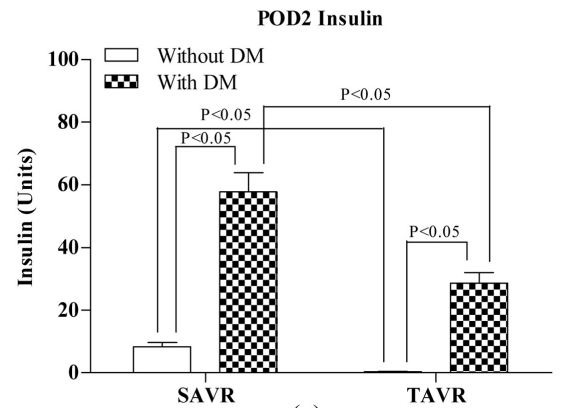

(c)

POD4 Insulin

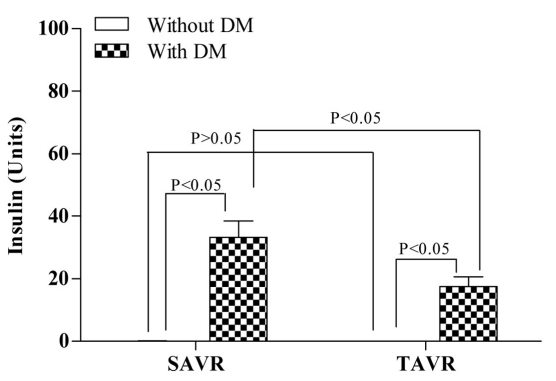

(e)

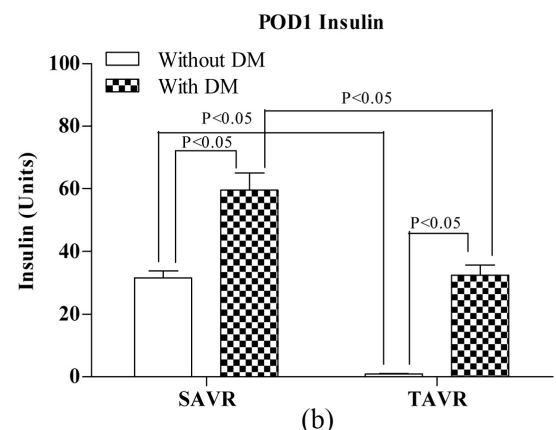

(b)

POD3 Insulin

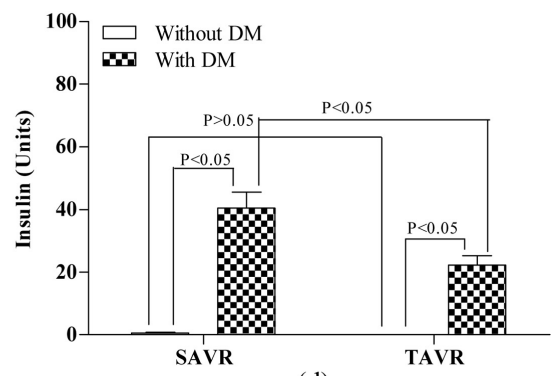

(d)

Figure 2. The amount of Insulin usage from POD0 to POD4. SAVR w/o DM: $n=128$; SAVR w DM: $\mathrm{n}=58$; TAVR w/o DM: $\mathrm{n}=173$; TAVR $\mathrm{w}$ DM: $\mathrm{n}=146$. Data presented as Mean \pm SME. $P$ values presented as in panel. 
Table 2. Percentage of patients using (CII + SC) insulin by post-procedure days (POD).

\begin{tabular}{lcccc}
\hline & SAVR w DM & SAVR w/o DM & TAVR w DM & TAVR w/o DM \\
\hline POD1 & $95 \%$ & $84 \%$ & $81 \%$ & $13 \%$ \\
POD2 & $98 \%$ & $46 \%$ & $70 \%$ & $4 \%$ \\
POD3 & $93 \%$ & $9 \%$ & $70 \%$ & $3 \%$ \\
POD4 & $88 \%$ & $3 \%$ & $59 \%$ & $0 \%$ \\
\hline
\end{tabular}

(SAVR and TAVR) and between patients with or without DM respectively (Figures 2(b)-(e)). This trend was similar for length of insulin infusion (Figure 4).

\section{2) Blood Glucose Ranges:}

Average BG was higher in patients with DM than those w/o DM in both TAVR and SAVR patients and the ranges are shown in Figure 3.

In the SAVR group (Figure 3(a) \& Figure 3(b)), the ranges of BG were greater than the TAVR group (Figure $3(\mathrm{c})$ \& Figure 3(d)). Patients without $\mathrm{DM}$, in both SAVR and TAVR groups, have a narrow range of BG which trended down on subsequent POD (Figure 3(a) \& Figure 3(c)). Patients with DM in both SAVR and TAVR groups have wide and similar BG ranges (Figure 3(b) \& Figure 3(d)). These phenomena parallel insulin dosing and insulin usage.

\section{3) Insulin Infusion Transition to Subcutaneous Time:}

Transition time of insulin infusion to subcutaneous insulin was significantly lower in the TAVR group compared to SAVR, $\mathrm{P}<0.05$ (Figure 5). Patients with $\mathrm{DM}$ had a longer time to transition to subcutaneous insulin $(\mathrm{P}<0.05$ Figure 5$)$. For TAVR with DM, the transition length was $1.02 \pm 0.09$ days, TAVR without DM was $0.12 \pm 0.03$ day, SAVR with DM was $2.35 \pm 0.10$ day and SAVR without DM was $1.33 \pm 0.07$ day.

4) Separating DM patients by A1c for Insulin Management of both Procedure:

In the SAVR group, for patients utilizing the same insulin infusion protocol and BG targets (Figure 5(b)) we observed that insulin requirements were associated with their preoperative $\mathrm{HbAlc}$ levels. Patients with $\mathrm{HbA1c}>6.5 \%$ needed relatively higher insulin infusion rates compared to those with $\mathrm{HbAlc}<6.5 \%$ (Figure 5(c)). However, for SAVRs, there was no difference in the insulin infusion duration and transition to subcutaneous insulin based on HbAlc (Figure $5(d))$.

Within the TAVR group for DM patients, insulin infusion requirements and infusion lengths and transition time all paralleled $\mathrm{HbAlc}$ levels. Patients with $\mathrm{HbA1c}<6.5 \%$ required lower doses and days on insulin infusion and had shorter times to transition off the drip (Figure 6(d)), $\mathrm{P}<0.01$.

5) The Aassociation of Insulin Infusion, A1c and Preoperative BG Medications for TAVR patient:

On further analysis, in patients undergoing TAVR procedures with DM there was an association of insulin drip initiation with HbAlc and DM treatment 
Glucose ranges: SAVR without DM

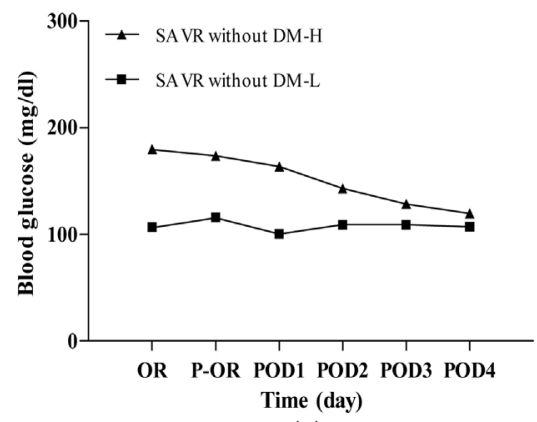

(a)

Glucose ranges: TAVR without DM

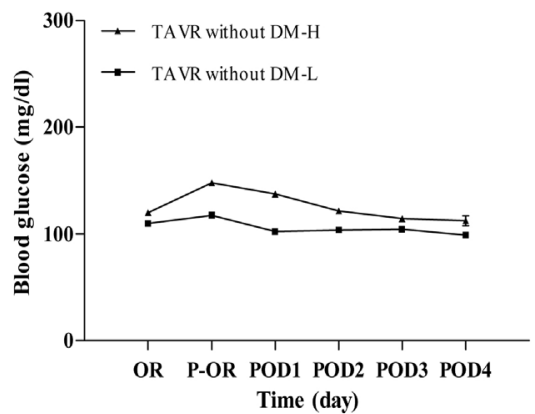

(c)

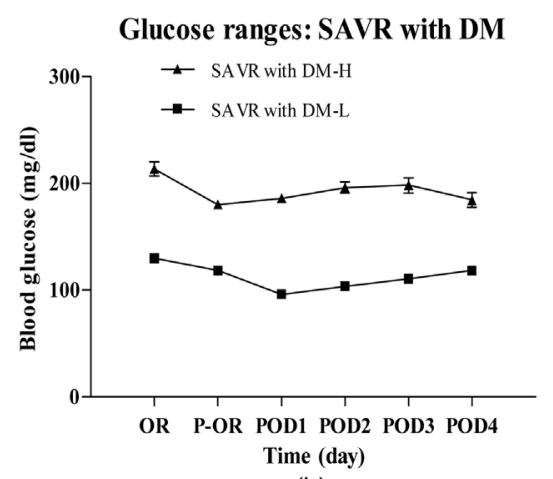

(b)

Glucose ranges: TAVR with DM

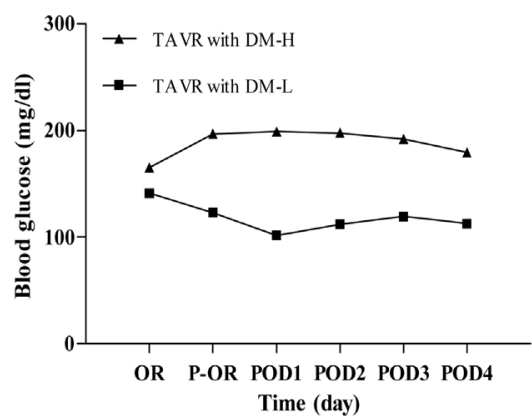

(d)

Figure 3. Average blood glucose ranges: $\mathrm{H}-$ the highest blood glucose levels on daily monitoring. L-the lowest blood glucose levels on daily monitoring. SAVR w/o DM: $\mathrm{n}=$ 128; SAVR w DM: $\mathrm{n}=58$; TAVR w/o DM: $\mathrm{n}=173$; TAVR w DM: $\mathrm{n}=146$. Data presented as Mean \pm SME.

\section{Transition time}

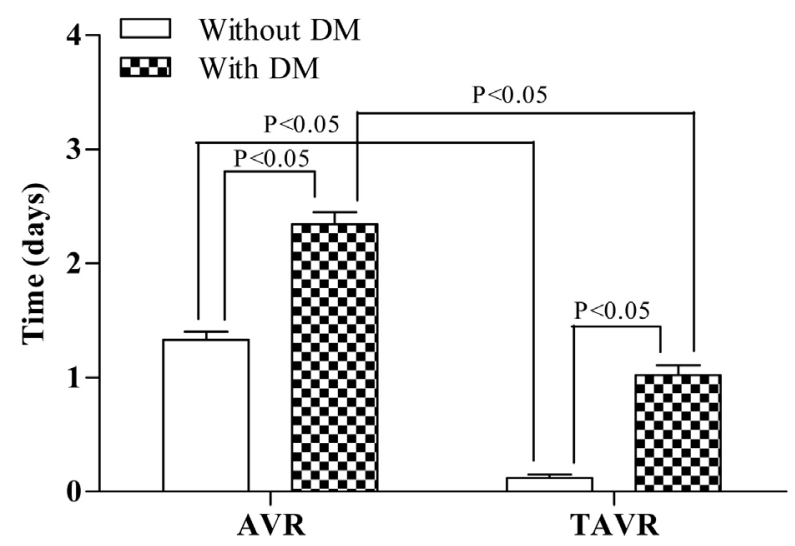

Figure 4. Transition time required from Insulin infusion to insulin subcutaneous injection. SAVR w/o DM: $n=128$; SAVR w DM: $\mathrm{n}=58$; TAVR w/o DM: $\mathrm{n}=173$; TAVR w DM: $\mathrm{n}=146$. Data presented as Mean \pm SME. One way ANOV analysis plus Tukey's Multiple Comparison Test performed. $\mathrm{P}$ value presented as in panel.

prior to procedure (Shown in Table 3). If A1c was lower than $6.5 \%$ in patients with DM controlled with diet, no insulin drip was required peri-TAVR. For 
HbA1c

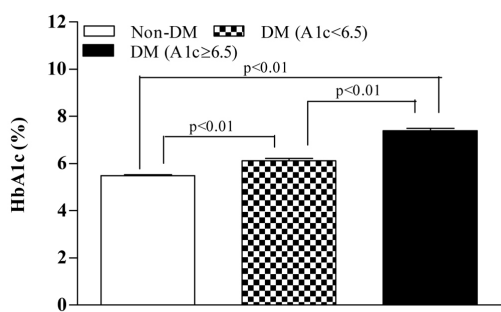

(a)

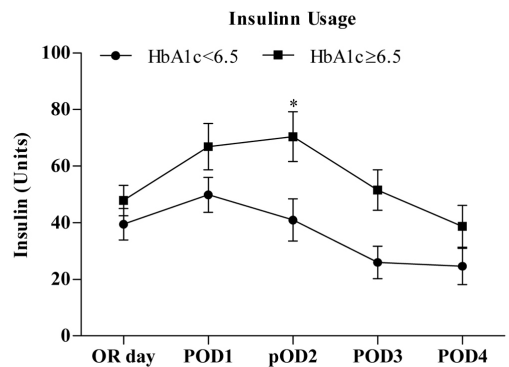

(c)
Glucose ranges: SAVR with DM

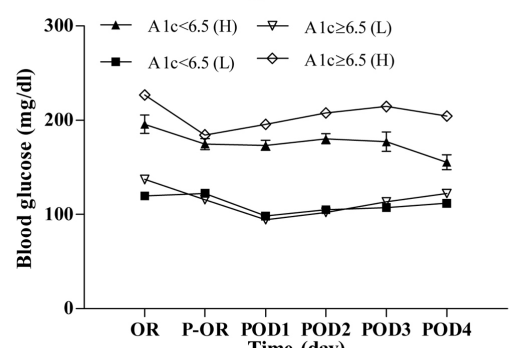

(b)

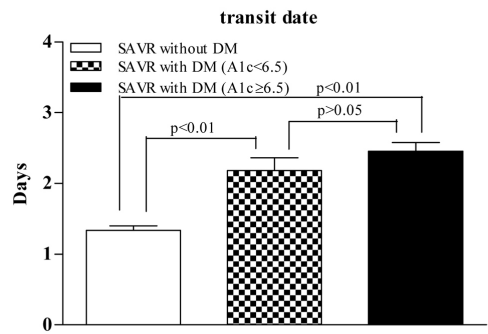

(d)

Figure 5. SAVR with DM and different HbAlc level, patient's blood glucose control, insulin requirements and insulin infusion lengths during perioperative period. Data presented as Mean \pm SEM. SAVR $w$ DM: $n=58$; SAVR $w$ DM $(A 1 c<6.5 \%): n=25$; SAVR $\mathrm{w}$ DM $(\mathrm{A} 1 \mathrm{c} \geq 6.5 \%): \mathrm{n}=33$. For panel $\mathrm{A}$ and $\mathrm{D}$, one-way ANOVA and Turkey's multiple tests employed for statistical analysis. For panel C, two-way ANOVA analysis employed.

HbA1c

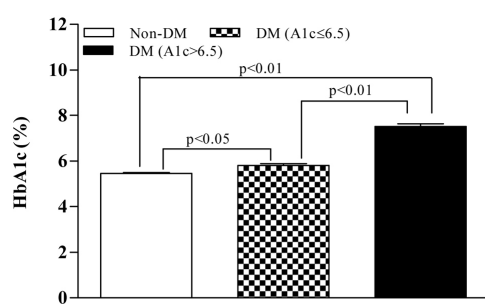

(a)

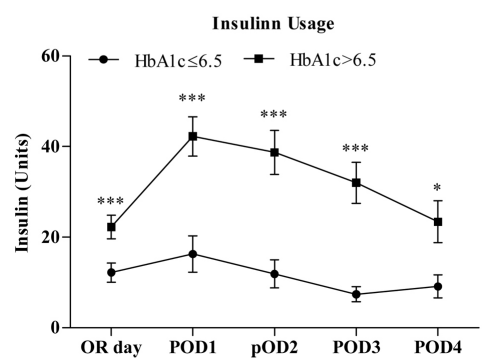

(c)
Glucose ranges: TAVR with DM

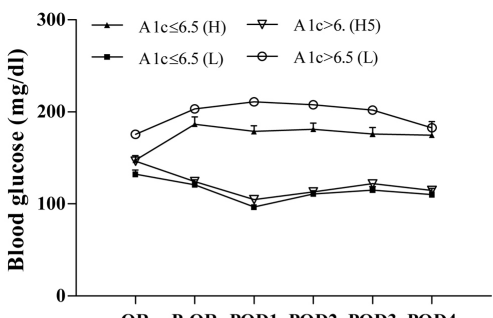

OR P-OR POD1 POD2 POD3 POD4 Time (day)

(b)

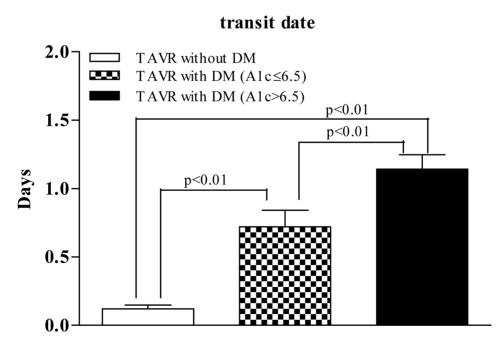

(d)

Figure 6. Blood glucose control for patients with diabetes having TAVR procedure during hospital stay. TAVR w DM divided into two groups according to the HbA1c level before procedure: $\mathrm{HbA} 1 \mathrm{c}<6.5 \%$ and $\mathrm{HbA} 1 \mathrm{c}>6.5 \%$. Data presented as Mean \pm SEM. SAVR w/o DM: $\mathrm{n}=128$; TAVR w DM (A1c <6.5\%): $\mathrm{n}=25$; TAVR w DM $(\mathrm{A} 1 \mathrm{c} \geq 6.5 \%): \mathrm{n}=33$. For panel A and D, one-way ANOVA and Turkey's multiple test employed for statistical analysis. For panel C, two-way ANOVA analysis employed. 
Table 3. Percentage of insulin infusion after TAVR procedure in patients with diabetes divided according to preoperative therapy. Data presented as percentage of patients having insulin infusion after TAVR.

\begin{tabular}{cccc}
\hline TAVR with DM & Diet Control & $\begin{array}{c}\text { Oral Medications } \\
\text { Control }\end{array}$ & Insulin Control \\
\hline $\begin{array}{c}\text { Total 146 patients } \\
\text { 62 patients Alc }<6.5 \% \text { with } \\
\text { insulin infusion \% }\end{array}$ & 48 & 40 & 58 \\
$\begin{array}{c}\text { 84 patients Alc } \geq 6.5 \% \text { with } \\
\text { insulin infusion \% }\end{array}$ & 0 & 50 & 90.91 \\
P Value & 33.33 & 68.75 & 78.13 \\
\hline
\end{tabular}

patients on oral medications for DM and $\mathrm{HbA} 1 \mathrm{c}<6.5 \%, 50 \%$ needed an insulin infusion. For patients with DM controlled with insulin and with A1c $<6.5 \%$, $90.9 \%$ needed an insulin infusion. If $\mathrm{A} 1 \mathrm{c} \geq 6.5 \%$, in the peri-TAVR procedure, $33.3 \%$ of diet-controlled patients, $68.8 \%$ on oral medications and $78.1 \%$ on insulin needed insulin infusion.

\section{6) The Hospital Length of Stay:}

Post-operative Length of Stay (LOS) was shorter with TAVR (5.3 \pm 0.31 days) compared with SAVR $(7.9 \pm 1.18$ days $)(\mathrm{P}<0.05)$. For the same procedure in the SAVR group, without DM, LOS was shorter than for those with DM (6.86 \pm 0.34 vs $8.97 \pm 0.84$ days, $\mathrm{P}<0.05)$. In TAVR patient there was no difference in LOS between those with or without DM (5.90 \pm 0.40 vs $4.73 \pm 0.22$ days) (Figure 7).

\section{Discussion}

In this retrospective review of patients undergoing SAVR and TAVR procedures for aortic stenosis, we found many differences in intra- and post-operative insulin requirements between groups which translated into different length and duration of the insulin infusion. These observations are important as they allow us to design appropriate BG management strategies for these groups. Patients undergoing a TAVR procedure had much lower insulin usage, a shorter transition time for insulin infusion, and shorter hospital stays compared to those who underwent SAVRs. Insulin usage specifically in patients without DM undergoing a TAVR procedure was the lowest between all the groups.

This finding is important as DM and AS are both prevalent in the elderly population and symptomatic AS is often seen together with DM [11]. Therefore, in the older TAVR population with DM there is less indication to recommend stringent glucose management strategies in the perioperative period. The TAVR procedure with its shorter OR time $(\mathrm{P}<0.05)$ and lower requirement of an insulin infusion for fewer perioperative days can allow for an early transition plan to subcutaneous insulin compared to the SAVR procedures (Figure 2).

These insulin requirements are probably related to stress-induced hyperglycemia, a transient phenomenon which is distinct from chronic glucose dysregulation of diabetes [3]. Studies have shown that stress hyperglycemia during 


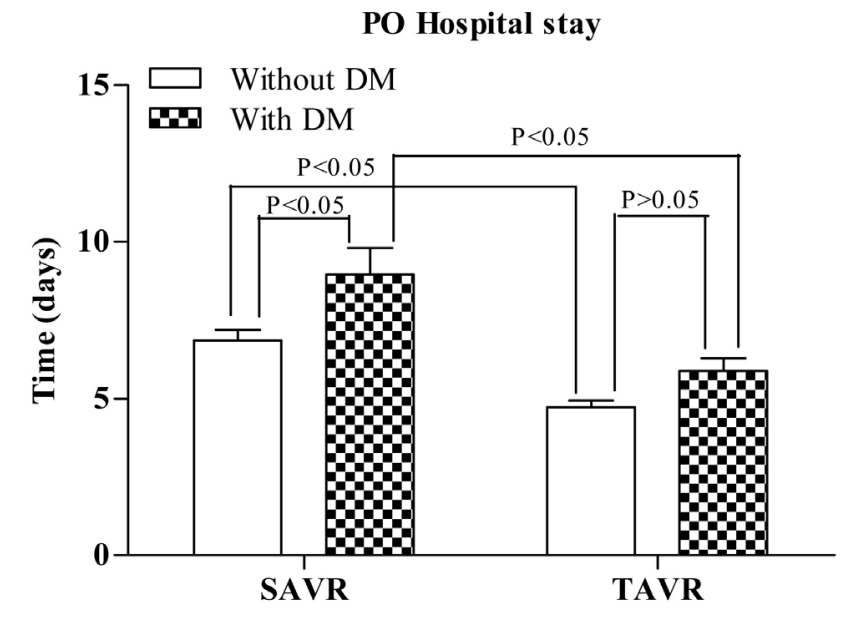

Figure 7. Post operation hospital stay time. SAVR w/o DM: $\mathrm{n}=$ 128; SAVR w DM (HbA1c: $\geq 6.5 \%$ vs $<6.5 \%) \mathrm{n}=58$; TAVR w/o DM: $\mathrm{n}=173$; TAVR w DM (HbA1c: $\geq 6.5 \%$ vs $<6.5 \%): \mathrm{n}=146$.

and after cardiac surgery occurs in patients both with and without diabetes, and is associated with severity of surgical trauma and pre-procedural BG control [12] [13] [14] [15]. This state typically persists until inflammatory mediators have decreased and the catabolic state subsides [16] [17]. Surgical procedures and hypothermia are known to induce intraoperative hyperglycemia [18] [19] by various endocrine, metabolic and immunological pathways. Additionally, volatile anesthetics inhibit secretion of insulin in response to glucose, and thus augment the stress-induced hyperglycemia [20]. The degree and duration of hyperglycemia post aortic valve surgery are not well understood but also reflect several patient factors including the patient's insulin resistance, medications, nutrition and activity. The SAVR group has a longer surgical procedure with hypothermia, both of which are associated with stress hyperglycemia. This would account for higher insulin infusion rates and longer postoperative infusion durations to control hyperglycemia to similar goals as the TAVR group. Within the same procedure, the severity of surgical stress is similar but patients' baseline insulin resistance, weight, age, diabetes duration and their control are also important factors. This is reflected by SAVR patients having similar OR times $(\mathrm{P}>0.05)$ but with significantly different insulin usages between patient with or without DM. Patients in the DM subgroup needed higher insulin infusion rates, with longer time to transition to subcutaneous insulin after the procedure, compared to patients without DM.

Within the TAVR group, postoperative hyperglycemia was uncommon in the patients without DM even though severity of surgical stress was similar to those with DM. The mini surgical incision and procedure induce a lower level of hyperglycemia. In DM, patient factors were important and if A1c was controlled to lower than $6.5 \%$ by diet, insulin requirement was negligible. This was similar to a study by Tanaka [20] in which patients without DM having mini-invasive procedure did not require postoperative hyperglycemia management. This stra- 
tification almost accounts for individualized insulin therapy in TAVR patients based on their level of insulin resistance and BG control.

Insulin is the most error prone medication in the hospital and hypoglycemia is related to insulin use and is associated with poor outcomes [21]. Utilizing this data, we can minimize insulin usage in low hyperglycemia risk, elderly and vulnerable TAVR populations.

Following cardiac surgery, patients with DM have been shown to suffer higher rates of renal dysfunction, need for blood transfusions and lung complications. DM patients undergoing TAVR have been shown to have fewer complications than DM patients undergoing SAVR [22]. Berkovitch et al. report that patients with diabetes undergoing TAVR seem to have favorable outcomes with similar short and mid-term mortality rates compared to those without DM. Patients with diabetes do suffer increased rates of AKI after SAVR or TAVR procedures [7]. As patients with insulin-requiring DM have greater incidence of renal insufficiency at baseline, and therefore a higher risk of renal decline and hypoglycemia,

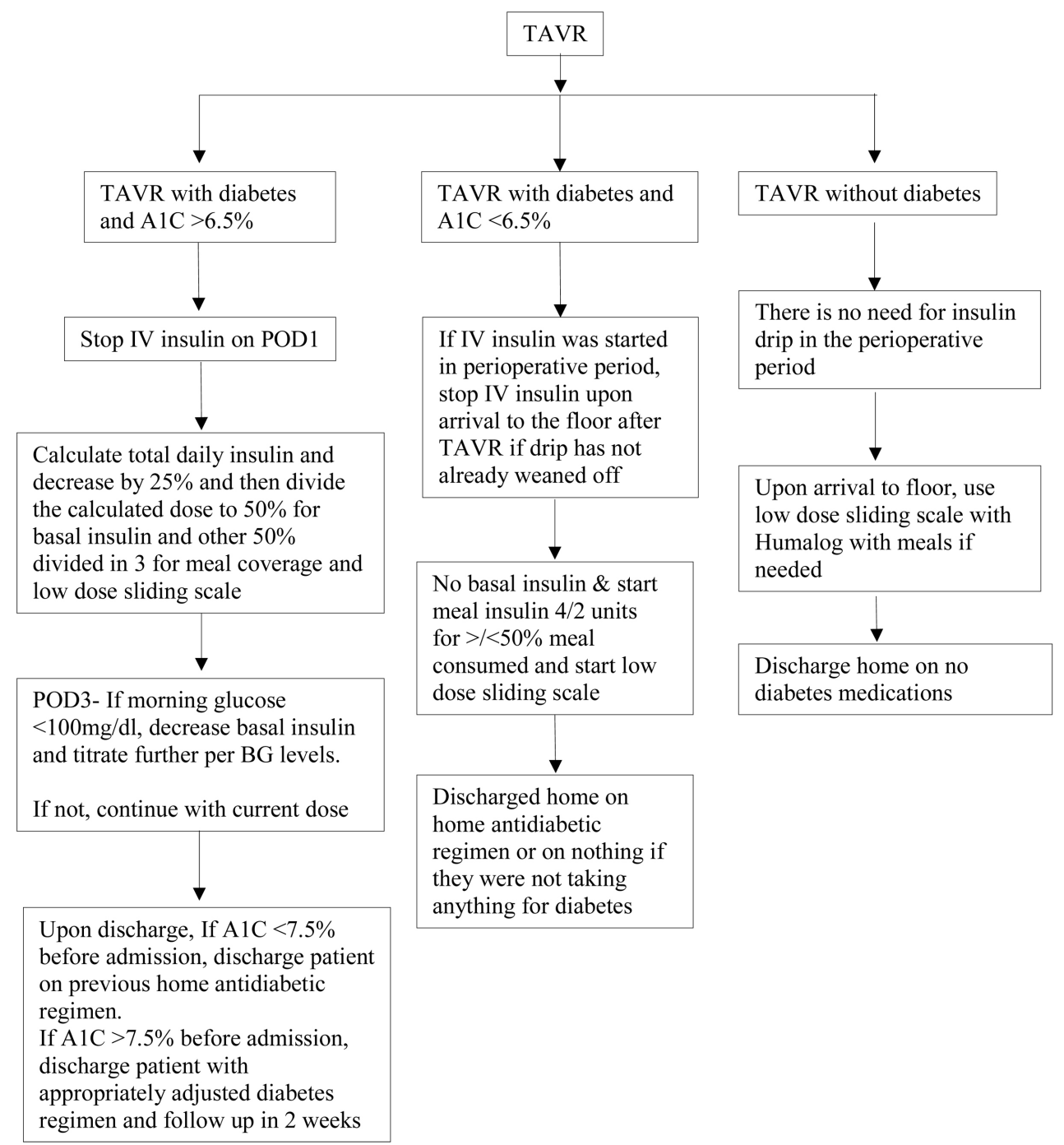

Figure 8. Algorithm of blood glucose management in TAVR patients. 
appropriate use of insulin can be designed utilizing our data.

This study has several limitations. First, the patient numbers are moderate and a larger cohort can provide a more robust conclusion. Second, the general follow-up duration is short, from the operation day to POD 4. Studies with longer follow-up duration would be helpful. Third, the study did not consider the duration of DM which can affect insulin resistance and outcomes. Due to the criteria of procedure selection, the average age of the patient having TAVR was higher than patient having SAVR, and other age-related comorbidities may be confounders. A future, large prospective evaluation with a predetermined insulin protocol separate for SAVR and TAVR patients could provide more robust information.

Our data allow us to propose an algorithm for post surgical management of BG in patients with aortic stenosis as summarized in Figure 8.

\section{Conclusion}

In conclusion, this study is the first to evaluate the differences in insulin requirement between SAVR and TAVR patients. The longer surgical duration and hypothermic circulatory arrest in patients undergoing SAVR lead to significant increase in amount and duration of insulin use in patients with and without diabetes. Insulin is only required in TAVR patients with diabetes but at lower doses due to less surgical stress. As TAVR procedures become more widespread, this data provides insight to develop personalized algorithms to simplify BG management in the AS population.

\section{Acknowledgements}

We thank the cardiac surgery department, cardiovascular ICU unit of Michigan Medicine, and Amy Geltz and Jeremy J. Wolverton from cardiac surgery department, Michigan Medicine for helping with data.

\section{Conflicts of Interest}

The authors declare no conflicts of interest regarding the publication of this paper.

\section{References}

[1] Ho, C., Cimon, K. and Rabb, D. (2013) CADTH Rapid Response Reports. In: Transcatheter Aortic Valve Replacement in Severe Aortic Stenosis. A Review of Comparative Durability and Clinical Effectiveness beyond 12 Months, Canadian Agency for Drugs and Technologies in Health, Ottawa.

[2] https://newheartvalve.com/ https://newheartvalve.com/hcp/about-aortic-stenosis/

[3] Dungan, K.M., Braithwaite, S.S. and Preiser, J.C. (2009) Stress Hyperglycaemia. The Lancet, 373, 1798-807. https://doi.org/10.1016/S0140-6736(09)60553-5

[4] Jarvela, K.M., Khan, N.K., Loisa, E.L., Sutinen, J.A., Laurikka, J.O. and Khan, J.A. (2017) Hyperglycemic Episodes Are Associated with Postoperative Infections after 
Cardiac Surgery. Scandinavian Journal of Surgery, 107, 138-144.

https://doi.org/10.1177/1457496917731190

[5] Umpierrez, G.E., Isaacs, S.D., Bazargan, N., You, X., Thaler, L.M. and Kitabchi, A.E. (2002) Hyperglycemia: An Independent Marker of in-Hospital Mortality in Patients with Undiagnosed Diabetes. The Journal of Clinical Endocrinology \& Metabolism, 87, 978-982. https://doi.org/10.1210/jcem.87.3.8341

[6] Greco, G., et al. (2016) Diabetes and the Association of Postoperative Hyperglycemia with Clinical and Economic Outcomes in Cardiac Surgery. Diabetes Care, 39, 408-417. https://doi.org/10.2337/dc15-1817

[7] Berkovitch, A., et al. (2015) Clinical Impact of Diabetes Mellitus in Patients Undergoing Transcatheter Aortic Valve Replacement. Cardiovascular Diabetology, 14, 131. https://doi.org/10.1186/s12933-015-0291-3

[8] Chorin, E., et al. (2015) Impact of Diabetes Mellitus and Hemoglobin A1C on Outcome after Transcatheter Aortic Valve Implantation. The American Journal of Cardiology, 116, 1898-1903. https://doi.org/10.1016/j.amjcard.2015.09.032

[9] Mendez-Bailon, M., et al. (2017) Transcatheter Aortic Valve Implantation and Surgical Aortic Valve Replacement among Hospitalized Patients with and without Type 2 Diabetes Mellitus in Spain (2014-2015). Cardiovascular Diabetology, 16, 144. https://doi.org/10.1186/s12933-017-0631-6

[10] Abramowitz, Y., et al. (2016) Impact of Diabetes Mellitus on Outcomes after Transcatheter Aortic Valve Implantation. The American Journal of Cardiology, 117, 1636-1642. https://doi.org/10.1016/j.amjcard.2016.02.040

[11] Falcão-Pires, I., et al. (2011) Diabetes Mellitus Worsens Diastolic Left Ventricular Dysfunction in Aortic Stenosis through Altered Myocardial Structure and Cardiomyocyte Stiffness. Circulation, 124, 1151-1159. https://doi.org/10.1161/CIRCULATIONAHA.111.025270

[12] Ata, A., Lee, J., Bestle, S.L., Desemone, J. and Stain, S.C. (2010) Postoperative Hyperglycemia and Surgical Site Infection in General Surgery Patients. Archives of Surgery, 145, 858-864. https://doi.org/10.1001/archsurg.2010.179

[13] Desai, S.P., et al. (2012) Strict versus Liberal Target Range for Perioperative Glucose in Patients Undergoing Coronary Artery Bypass Grafting: A Prospective Randomized Controlled Trial. The Journal of Thoracic and Cardiovascular Surgery, 143, 318-325. https://doi.org/10.1016/j.jtcvs.2011.10.070

[14] Lazar, H.L., et al. (2009) The Society of Thoracic Surgeons Practice Guideline Series: Blood Glucose Management during Adult Cardiac Surgery. The Annals of Thoracic Surgery, 87, 663-669. https://doi.org/10.1016/j.athoracsur.2008.11.011

[15] Breithaupt, T. (2010) Postoperative Glycemic Control in Cardiac Surgery Patients. Baylor University Medical Center Proceedings, 23, 79-82. https://doi.org/10.1080/08998280.2010.11928586

[16] McCowen, K.C., Malhotra, A. and Bistrian, B.R. (2001) Stress-Induced Hyperglycemia. Critical Care Clinics, 17, 107-124. https://doi.org/10.1016/S0749-0704(05)70154-8

[17] Epstein, J. and Breslow, M.J. (1999) The Stress Response of Critical Illness. Critical Care Clinics, 15, 17-33. https://doi.org/10.1016/S0749-0704(05)70037-3

[18] McCavert, M., Mone, F., Dooher, M., Brown, R. and O’Donnell, M.E. (2010) Peri-Operative Blood Glucose Management in General Surgery-A Potential Element for Improved Diabetic Patient Outcomes-An Observational Cohort Study. International Journal of Surgery, 8, 494-498. https://doi.org/10.1016/j.ijsu.2010.06.017

[19] Sudhakaran, S. and Surani, S.R. (2015) Guidelines for Perioperative Management of 
the Diabetic Patient. Surgery Research and Practice, 2015, Article ID: 284063. https://doi.org/10.1155/2015/284063

[20] Tanaka, T., Nabatame, H. and Tanifuji, Y. (2005) Insulin Secretion and Glucose Utilization Are Impaired under General Anesthesia with Sevoflurane as well as Isoflurane in a Concentration-Independent Manner. Journal of Anesthesia, 19, 277-281. https://doi.org/10.1007/s00540-005-0341-1

[21] Kalra, S., et al. (2013) Hypoglycemia: The Neglected Complication. Indian Journal of Endocrinology and Metabolism, 17, 819-834. https://doi.org/10.4103/2230-8210.117219

[22] Lindman, B.R., et al. (2014) Transcatheter versus Surgical Aortic Valve Replacement in Patients with Diabetes and Severe Aortic Stenosis at High Risk for Surgery: An Analysis of the PARTNER Trial (Placement of Aortic Transcatheter Valve). Journal of the American College of Cardiology, 63, 1090-1099.

https://doi.org/10.1016/j.jacc.2013.10.057

\section{Supplement}

Table S1. Average insulin usage in on POD1-4. Data presented as mean \pm SME.

\begin{tabular}{cccc}
\hline & \multicolumn{2}{c}{ Without DM } & With DM \\
\cline { 2 - 4 } & & $\begin{array}{c}\text { Insulin dose units } \\
\text { Mean } \pm \text { SEM }\end{array}$ & $\begin{array}{c}\text { Insulin dose units } \\
\text { Mean } \pm \text { SEM }\end{array}$ \\
POD1 & SAVR & $31.65 \pm 2.22$ & $59.53 \pm 5.486$ \\
& TAVR & $0.9 \pm 0.2618$ & $32.46 \pm 3.248$ \\
POD2 & SAVR & $8.403 \pm 1.291$ & $57.7 \pm 6.2$ \\
& TAVR & $0.3754 \pm 0.2033$ & $28.63 \pm 3.407$ \\
POD3 & SAVR & $0.6411 \pm 0.2927$ & $40.51 \pm 5.014$ \\
& TAVR & $0.08276 \pm 0.04745$ & $22.32 \pm 3.035$ \\
POD4 & SAVR & $0.07018 \pm 0.04266$ & $33.25 \pm 5.213$ \\
& TAVR & $0.000909 \pm 0.000909$ & $17.51 \pm 3.002$ \\
\hline
\end{tabular}

\section{Abbreviation}

SAVR: Surgical Aortic Valve Replacement

TAVR: Trans-Catheter Aortic Valve Replacement

AS: Aortic Stenosis

BG: Blood Glucose

BGR: Blood Glucose Range

DMH: Diabetes Mellitus History

HbAlc: Hemoglobin A1C

ICU: Intensive Care Unit

LOS: Length of Stay

POD: Post Operation Days 